\title{
Made you blink! Contingent attentional capture produces a spatial blink
}

\author{
CHARLES L. FOLK \\ Villanova University, Villanova, Pennsylvania \\ and \\ ANDREW B. LEBER and HOWARD E. EGETH \\ Johns Hopkins University, Baltimore, Maryland
}

\begin{abstract}
Previous studies have shown that the capture of attention by an irrelevant stimulus can be eliminated by foreknowledge of the spatial location of the relevant target stimulus. To explore whether spatial certainty is sufficient to eliminate capture, four experiments are reported in which the spatial location of the target is certain but the temporal position is uncertain. Subjects viewed a central rapid serial visual presentation stream in which a target letter was defined by a particular color (e.g., red). On critical trials, irrelevant color singletons appeared in the periphery. In Experiments 1 and 2, peripheral singletons produced a decrement in central target identification that was contingent on the match between the singleton color and the target color. Experiments 3 and 4 provided evidence that this decrement reflected a shift of spatial attention to the location of the distractor. The results suggest that spatial certainty is not sufficient to eliminate attentional capture and that attentional capture can result in a spatial "blink" that is conditional on top-down attentional control settings.
\end{abstract}

There is growing evidence that the efficient allocation of visual selective attention involves a delicate interplay between the properties of the stimulus itself and the behavioral goals of the observer. Although salient stimulus properties (e.g., abrupt onset, movement, discontinuities in color, etc.) have been shown to attract attention involuntarily (Remington, Johnston, \& Yantis, 1992; Theeuwes, 1992, 1994; Yantis \& Jonides, 1984, 1990), there is also evidence that such instances of attentional capture can be modulated by top-down attentional set (Bacon \& Egeth, 1994; Folk \& Remington, 1998; Folk, Remington, \& Johnston, 1992; Folk, Remington, \& Wright, 1994; Gibson \& Kelsey, 1998; Yantis \& Jonides, 1990). For example, using a spatial-cuing task, Folk et al. (1992) found that the capture of spatial attention by irrelevant abrupt-onset precues was contingent on the attentional set of the observer. Specifically, when the target was an abrupt onset, irrelevant abrupt-onset precues produced spatial cuing effects indicative of capture. When the target was defined by a discontinuity in color (i.e., a color singleton), however, the very same abrupt-onset precues no longer produced evidence of capture. Similarly, Folk and Remington (1998) found that color singleton precues produced evidence of

This research was supported in part by National Science Foundation Grant BCS-9817673 to C.L.F. and in part by National Institute of Mental Health Grant MH57388 to H.E.E. We thank Vince Di Lollo, Anne Hillstrom, Matt Peterson, Eric Ruthruff, Jim Juola, and an anonymous reviewer for helpful comments on earlier versions of this work. Correspondence should be addressed to C. L. Folk, Department of Psychology, Villanova University, Villanova, PA 19085 (e-mail: charles.folk@villanova.edu). attentional capture, but only when the target was a singleton of the same color. On the basis of these and other results, Folk and colleagues proposed the contingent involuntary orienting hypothesis, which states that a given stimulus property will capture attention only to the extent that it matches top-down attentional control settings. These control settings are assumed to be a function of the current behavioral goals of the observer, such as searching for a red letter.

Another form of top-down control over attentional capture involves the degree to which attention is spatially focused prior to the presentation of a salient irrelevant stimulus. Yantis and Jonides (1990) found that when the location of a target letter in a visual search task was uncertain, the presence of an irrelevant abrupt-onset letter produced evidence of attentional capture. However, when subjects were given a $100 \%$ valid precue indicating the subsequent location of a target, capture effects were eliminated. Similar results were reported by Theeuwes (1991), who systematically varied the stimulus onset asynchrony (SOA) between the presentation of a precue (a $100 \%$ valid, centrally presented arrow) and a target, as well as the SOA between an irrelevant onset (and offset) and a target. When the location precue preceded the target by 300 or $600 \mathrm{msec}$, irrelevant onsets (and offsets) appearing at nontarget locations had no effect on responses to the target, regardless of whether the irrelevant onset (offset) appeared before, simultaneously with, or after target presentation. These results support the widely held belief that when spatial attention is in a highly focused state, salient stimuli (such as abrupt onsets) are no longer capable of capturing spatial attention. ${ }^{1}$ 
A potentially important aspect of these studies, however, is that the use of a $100 \%$ valid spatial precue not only eliminated uncertainty about the target location, but also eliminated any uncertainty about which object in the display was the target. That is, on any given trial, only one object ever occurred at the cued location. One could imagine a situation in which the target location is known but multiple objects appear at that location, producing uncertainty with regard to which object is the target. Consider, for example, a typical rapid serial visual presentation (RSVP) experiment in which a stream of letters appears at fixation. Although all of the letters in the stream are spatially attended, there is still uncertainty about which is the target. This uncertainty is typically resolved through an additional act of nonspatial selection based on properties such as color or shape (e.g., report the red letter in the stream). It is unknown whether the elimination of attentional capture by events outside the focus of attention still holds when there remains uncertainty about which object within the focus of attention is the target. In other words, is the focusing of attention sufficient to override attentional capture?

Furthermore, it is likely that the nonspatial selection of a target involves the establishment of top-down attentional control settings for the defining property of the target. For example, determining the identity of a red letter in a temporal sequence of white letters would presumably require an attentional control setting for the color red. A second issue, therefore, concerns the extent to which this attentional control setting for nonspatial selection would influence the allocation of attention in space. Specifically, what effect would an irrelevant distractor have if it appeared outside the focus of spatial attention but matched the attentional control setting (e.g., red) for nonspatial selection from a temporal sequence? If the focusing of spatial attention is sufficient to eliminate capture, peripheral events should not interfere with the identification of targets at the focused location. If, on the other hand, attentional control settings for nonspatial selection influence the allocation of spatial attention, then, consistent with the contingent involuntary orienting hypothesis, we might expect an irrelevant stimulus that matches the attentional control setting to capture attention even if the stimulus occurs outside the focus of attention. ${ }^{2}$

To address these issues, we used a variant of the RSVP paradigm that has been used to study a phenomenon known as the attentional blink (AB). In the typical AB task, a rapid series of stimuli is presented at fixation, and either one or two targets can appear within the stream (see, e.g., Broadbent \& Broadbent, 1987; Chun \& Potter, 1995; Raymond, Shapiro, \& Arnell, 1992, 1995). The AB refers to a decrement in the detection or identification of the second target (T2) when it occurs soon after the presentation of a first target (T1), to which a response is required. Although the precise mechanism is still under debate, the decrement in $\mathrm{T} 2$ processing is generally assumed to be a by-product of attentional selection of T1. In our task, subjects were required to monitor a centrally presented stream of letters for a target letter of a particular color and to report the identity of that letter. It was assumed that this task would require attention to be tightly focused at fixation. However, instead of an additional target in the stream, a task-irrelevant, peripheral distractor was presented at different temporal positions relative to the target. Since the most interesting data derive from the conditions in which the peripheral distractor preceded the central target, one can think of that distractor as playing the role of $\mathrm{T} 1$ in the standard $\mathrm{AB}$ paradigm. However, it is important to keep in mind that there was no task associated with that stimulus; it was an irrelevant distractor. The critical manipulation was whether this distractor shared the color that defined the central target or not. We reasoned that if a peripheral distractor matching the color of the central target captures attention, then even under the focused attentional state required by the central stream, a decrement in the identification of the centrally presented target should obtain.

\section{EXPERIMENT 1}

\section{Method}

Subjects. Thirty-three undergraduate students from Villanova University participated in a single, 50-min experimental session for credit toward fulfillment of a class research requirement. All had self-reported normal or corrected-to-normal visual acuity and color vision.

Apparatus and Stimuli. The stimuli were presented on a Sony Trinitron Multiscan 500 PS 21-in. monitor, driven by a Hewlett Packard Vectra VL computer. The subjects viewed the monitor in a dimly lit room at a distance of approximately $50 \mathrm{~cm}$.

The stimuli consisted of a series of single letters presented in the center of the computer screen. Letters measured $1.3^{\circ}$ in height and $1.2^{\circ}$ in width, with a stroke width of $0.3^{\circ}$. One letter in the sequence was red or green, and the remaining letters were gray against the dark background of the monitor. On trials containing a distractor, one of the items in the series consisted of a central letter surrounded by four \#s whose inner edges appeared $5.2^{\circ}$ above, below, right, and left of the center of the letter. Depending on the distractor condition, either all the \#s were gray, or three were gray and one was red or green.

Design and Procedure. Each trial of the experiment began with a 500-msec presentation of a white fixation cross in the middle of the screen, followed after $200 \mathrm{msec}$ by the sequential presentation of 15 letters. Each letter in the sequence was presented for $42 \mathrm{msec}$, followed by a $42-\mathrm{msec}$ blank interval, yielding an SOA of $84 \mathrm{msec}$ (see Figure 1). ${ }^{3}$ The letters on each trial were selected randomly without replacement from the English alphabet, with the exception of I, O, W, and Z. For 17 of the subjects, one of the letters in the sequence was red, and for the other 16 subjects one of the letters was green. For all the subjects, the remaining letters were gray. Across trials, the target appeared equally often in positions 8 through 12 of the letter sequence.

Each subject was presented with four different distractor conditions, mixed within blocks. In the no-distractor condition, each of the 15 frames in the trial series contained only the letter in the center of the screen. The remaining conditions differed in that, on one of the frames, distractors were presented in addition to the letter at fixation. In the four-gray distractor condition, one frame in the series contained a letter surrounded by four eccentric gray \#s, as was 


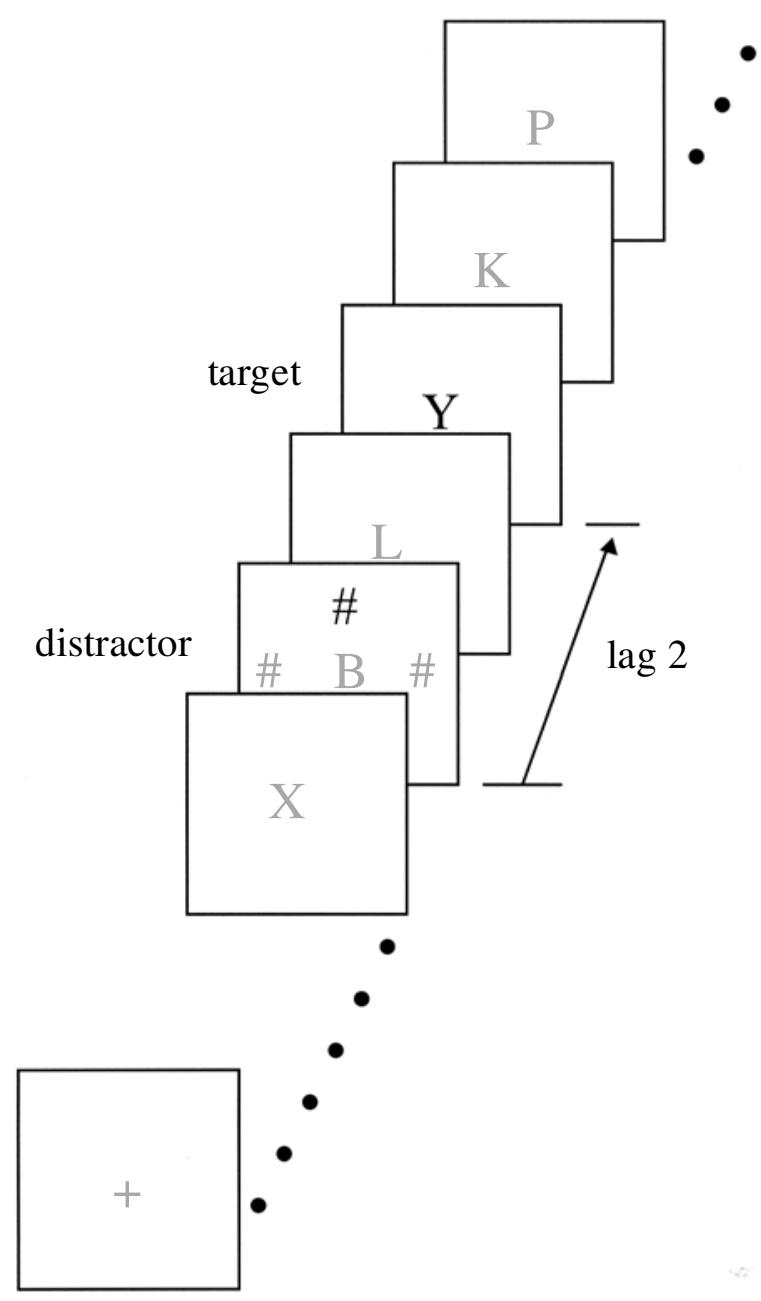

Figure 1. Representation of stimuli and sequence of events on a trial with a distractor-target lag of 2 . The characters printed in black were actually red or green (see the text).

described above. In the same-color distractor condition, one frame contained a letter surrounded by three gray \#s and one colored \# that matched the color of the target for which the subjects were searching. For example, if a subject was searching for a red target, a same-color distractor trial would consist of three gray \#s and one red \#. Finally, in the different-color distractor condition, one frame contained a letter surrounded by three gray \#s and one \# that was different from the color of the target. For example, if a subject was searching for a red target, a different-color distractor would consist of three gray \#s and one green \#. In the latter two conditions, the colored \# appeared equally often in the four possible locations.

The four distractor conditions were crossed with four temporal lags between the presentation of the distractor and the presentation of the target. The target could appear two frames after the distractor (lag 2), one frame after the distractor (lag 1), simultaneously with the distractor (lag 0), or one frame before the distractor (lag -1). Trials in the no-distractor condition were assigned a lag value, but the distractor was omitted from the sequence. Across trials, each distractor type appeared equally often at each possible lag.

The experiment consisted of 24 practice trials followed by a total of 320 trials, with a break after every 40 trials. The subjects received writ- ten and oral instructions regarding the nature of the stimuli and task. They were fully informed with respect to the various distractor conditions and were explicitly encouraged to ignore the distractor if possible. Each trial was initiated by a press of the space bar. After the trial sequence, the subjects were required to identify the target by typing in their response on the computer keyboard. They were instructed to guess if they were unsure of the identity of the target. They were then prompted by the computer to press the space bar to begin the next trial.

\section{Results}

The mean percentages of correct target identifications as a function of distractor condition and distractor-target lag are presented in Figure 2. These data were subjected to a mixed analysis of variance (ANOVA) with target color (red and green) as the single between-subjects variable and distractor condition (none, four gray, same color, and different color) and distractor-target lag $(-1,0,1$, and 2$)$ as the within-subjects variables. Neither the main effect of target color nor any of its interactions with other variables was significant. The analysis yielded significant main effects of distractor condition $\left[F(3,93)=23.53, M S_{\mathrm{e}}=\right.$ $113.97, p<.0001]$ and distractor-target lag $[F(3,93)=$ $\left.34.56, M S_{\mathrm{e}}=204.37, p<.0001\right]$ and a significant distractor condition $\times$ distractor-target lag interaction $[F(9,279)=$ $\left.14.25, M S_{\mathrm{e}}=117.57, p<.0001\right]$.

As is evident in the figure, the interaction was driven by the differential fall-off in performance for the same- and different-color conditions versus the no-distractor and gray distractor conditions at lags 1 and 2. This was confirmed by simple main effects analyses of distractor condition at each distractor-target lag. Only lags 1 and 2 produced a significant effect of distractor condition $[F(3,93)=34.56$, $M S_{\mathrm{e}}=103.7, p<.001$, and $F(3,93)=34.62, M S_{\mathrm{e}}=202.12$, $p<.0001$, respectively]. A Tukey test (alpha $=.05)$ at lag 1 revealed that only the mean for the same-color distractor differed significantly from that for the no-distractor condition. A similar analysis at lag 2 showed that the means for both the same- and the different-color distractor conditions differed significantly from both the no-distractor and the gray distractor conditions but did not differ significantly from one another.

\section{Discussion}

As is evident in Figure 2, the presence of an irrelevant, peripheral color singleton distractor produced a significant reduction in the accuracy of identifying a subsequent central target, relative to trials on which no distractor appeared, as well as to trials on which four gray distractors appeared. The relatively high performance in the latter condition confirms that the disruption by the color singleton distractors was not due to the mere presence of abrupt onsets in the periphery. (Note that the results in the gray distractor condition are also consistent with Yantis and Jonides's, 1990, finding that irrelevant abrupt onsets did not capture attention when subjects were spatially focused.) Together, these results suggest that even though the subjects were spatially focused on the central stream, an irrelevant color singleton in the periphery captured spatial attention in 


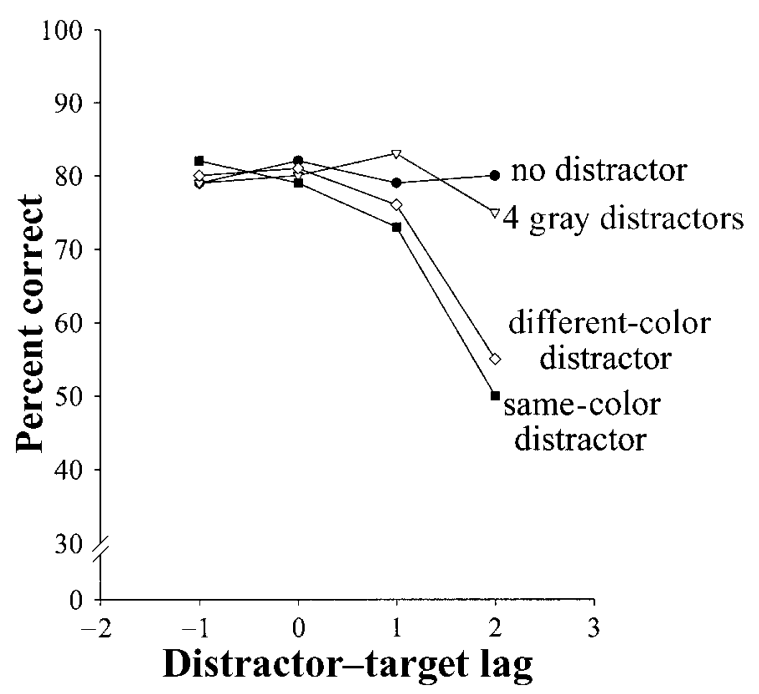

Figure 2. Mean percentages of correct target identification as a function of distractor condition and distractor-target lag in Experiment 1 . Negative lags reflect conditions in which the distractor appeared after the target.

such a way that it produced a decrement for centrally presented targets. Thus, the results provide evidence that the focusing of spatial attention is not sufficient to eliminate the capture of spatial attention by irrelevant stimuli.

The results also suggest that top-down attentional control settings have a powerful influence over the allocation of attention. In the present experiment, the spatial location of the target was known with absolute certainty, but the selection of the target from a distractor at that location could occur only through an attentional set for color. When the distractor matched this attentional set, it produced evidence of capture even though its presence and location were completely irrelevant to the task.

One puzzling aspect of the results, however, is the fact that both the same-color and the different-color distractors produced evidence of capture. If the subjects were really set for a specific color, why should a different-colored distractor produce capture? One possibility is that instead of an attentional set for a specific color, the subjects were set to monitor the central stream for a discontinuity in color, regardless of the value of that discontinuity. This notion is consistent with the distinction between feature search mode and singleton search mode proposed by Bacon and Egeth (1994) in the context of a visual search task (see also Pashler, 1988). They found that irrelevant singletons in a dimension different from the dimension defining the target (e.g., a color singleton distractor paired with a shape singleton target) produced evidence of capture. However, when the heterogeneity of the display along the target dimension was increased, singletons in the distractor dimension no longer produced evidence of capture. The authors argued that in the former case, subjects had adopted an attentional set for singletons in general, regardless of the dimensions over which the singletons were defined. In the latter case, the heterogeneity of objects along the target dimension forced subjects to adopt an attentional set for a particular feature value, thereby preventing capture by singletons in other dimensions.

It is possible, therefore, that the disruption by both same- and different-color distractor singletons reflects the adoption of a color singleton search mode in monitoring the central stream for a colored target. An alternative possibility is that the effect observed in this experiment is not related to attentional control settings at all but, instead, reflects capture by any color discontinuity that appears in the periphery. One straightforward means of distinguishing between these two accounts is to increase the color heterogeneity of the central stream in an effort to force subjects to adopt an attentional set for a particular color.

\section{EXPERIMENT 2}

Experiment 2 was conducted to test the hypothesis that the disruption of target identification by both same- and different-color distractors in Experiment 1 reflects the adoption of singleton search mode. The experiment was identical to Experiment 1, except that each of the nontarget letters in the central stream was one of four possible colors. This manipulation was designed to force subjects to adopt an attentional set for the particular target color. If attentional control settings were driving the capture of attention in Experiment 1, then in the present experiment, only distractors that match the target color should produce a decrement in target detection. If, on the other hand, any discontinuity in the periphery can capture attention, both same- and different-color distractors should once again produce the effect.

\section{Method}

Subjects. Thirty-two undergraduate students from Villanova University participated in a single, 50-min experimental session for credit toward fulfillment of a class research requirement. All had self-reported normal or corrected-to-normal visual acuity and color vision. None of the subjects had participated in Experiment 1. Sixteen of the subjects searched for a red target, and 16 searched for a green target.

Apparatus and Stimuli. The apparatus and stimuli were identical to those used in Experiment 1.

Design and Procedure. The design and procedure were identical to those in Experiment 1, with two exceptions. First, in contrast to Experiment 1, in which all the nontarget letters were gray, the color of each nontarget letter in the present experiment was chosen randomly from a set of four possible colors. For subjects searching for red targets, the colors were gray, blue, purple, and green. For subjects searching for green targets, the colors were gray, blue, purple, and red. Second, pilot work indicated that increasing the color heterogeneity of the central stream also increased the overall difficulty of the task. Therefore, the duration of each letter in the stream was increased from 42 to $56 \mathrm{msec}^{4}$ to bring overall performance to levels comparable to those in Experiment 1.

\section{Results}

The mean percentages of correct target identifications as a function of distractor condition and distractor-target lag are presented in Figure 3. The data were subjected to 


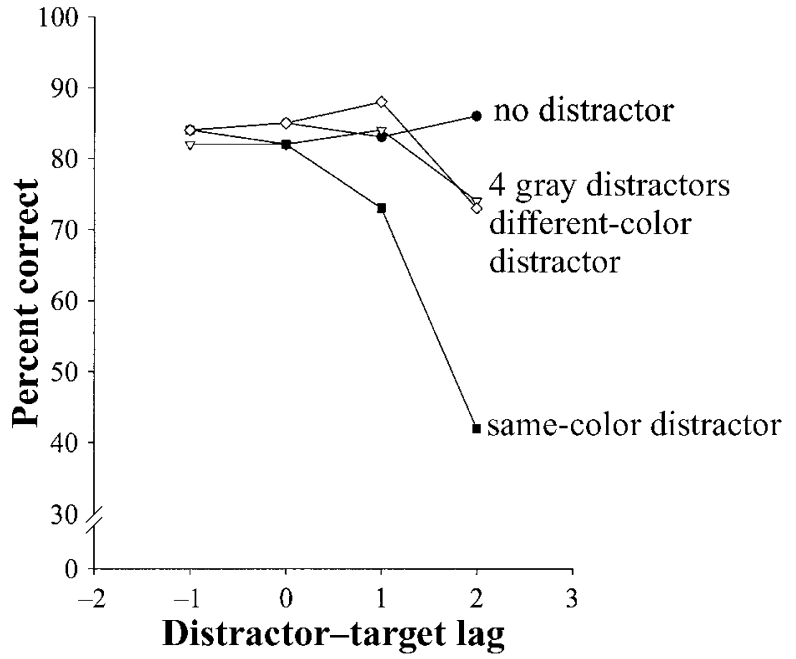

Figure 3. Mean percentages of correct target identification as a function of distractor condition and distractor-target lag in Experiment 2 . Negative lags reflect conditions in which the distractor appeared after the target.

the same analysis as that in Experiment 1. Once again, the main effect of target color was not significant, nor did it enter into interactions with any other variables. The main effects of distractor condition and distractor-target lag were significant $\left[F(3,90)=51.80, M S_{\mathrm{e}}=102.8, p<.0001\right.$, and $F(3,90)=37.10, M S_{\mathrm{e}}=197.4, p<.0001$, respectively], as was the distractor $\times$ lag interaction $[F(9,279)=$ 27.33, $\left.M S_{\mathrm{e}}=93.1, p<.0001\right]$.

As in Experiment 1, the figure suggests that the interaction is once again driven by performance at lags 1 and 2 . Simple main effects again yielded significanteffects of distractor condition only for lags 1 and $2[F(3,90)=16.52$, $M S_{\mathrm{e}}=84.4, p<.0001$, and $F(3,90)=75.72, M S_{\mathrm{e}}=150.9$, $p<.0001$, respectively]. A Tukey test (alpha $=.05)$ at lag 1 revealed that only the mean for the same-color distractor differed significantly from that for the no-distractor condition. At lag 2, all three distractor conditions differed significantly from the no-distractor condition. However, in contrast to Experiment 1, the mean for the different-color distractor condition differed significantly from that for the same-color distractor condition but did not differ from that for the four-gray distractor condition.

\section{Discussion}

The results of this experiment generally replicated those of Experiment 1, with one important exception. The decrement in target detection in the present experiment was specific to the conditions in which the distractor color matched the target color; the different-color distractor condition now produced little disruption in central target identification. These results confirm that the capture of spatial attention observed in the present experiments is driven by top-down attentional control settings and is not produced simply by any discontinuity in the periphery. In addition, the results suggest that the subjects in Experiment 1 had indeed adopted an attentional set for color discontinuity, consistent with Bacon and Egeth's (1994) work in the visual search domain.

\section{EXPERIMENT 3}

In the first two experiments, it was assumed that decrements in central target identification reflect a spatial blink in which access to the central target is disrupted by the involuntary orienting of spatial attention to an irrelevant peripheral location. It is possible, however, that the observed effects may not be spatial at all, reflecting instead a nonspatial effect similar to the traditional AB. In the standard paradigm, all stimuli are presented at the same location (usually fixation). Thus, the traditional $\mathrm{AB}$ reflects limits on attentional selection in time that are dissociable from effects related to the distribution of attention in space (e.g., Peterson \& Juola, 2000). In our first two experiments, distractors and targets were presented at different times and in different locations. Therefore, the observed effects could reflect a spatial blink (i.e., the capture of spatial attention), the traditional temporal blink, or both.

To address this issue, Experiment 2 was replicated with two critical changes. First, the four (identical) number-sign distractors were replaced with four (different) peripheral letters, one of which (i.e., the prime) was the same letter as the central target for that trial. Second, in the singleton distractor conditions, the location of the prime varied so that it appeared as the colored peripheral singleton on some trials and as one of the three gray nonsingletons on other trials. We reasoned that if spatial attention is drawn to the location of same-color peripheral singletons, the letter at that location should be identified. Furthermore, if the character at that location is the prime, we might expect central target identification to be enhanced, either through perceptual priming or through an advantageous guessing bias.

\section{Method}

Subjects. Twenty undergraduate students from Villanova University participated in this experiment. None had participated in the previous experiments. All had self-reported normal or corrected-tonormal visual acuity and color vision. Ten subjects searched for a red target, and 10 searched for a green target.

Apparatus and Stimuli. The apparatus and stimuli were identical to those used in Experiment 2; the subjects searched through a heterogeneous color stream for either a red or a green target letter. ${ }^{5}$

Design and Procedure. The design and procedure were identical to those in Experiment 2, with the following exceptions. First, the number signs used as distractors were replaced with letters of the same physical dimensions as those used in the central stream. One of these letters (the prime) was always the same as the central target for that trial. The three other letters were each chosen randomly without replacement, as in the previous experiments. In the samecolor and different-color distractor conditions, the prime appeared as the color singleton on one fourth of the trials. That is, across trials, the prime was no more likely to be the singleton than any other distractor letter. Thus, there was little incentive for the subjects to voluntarily shift spatial attention to the singleton. Second, in order to keep the total number of trials comparable to that used in the previ- 


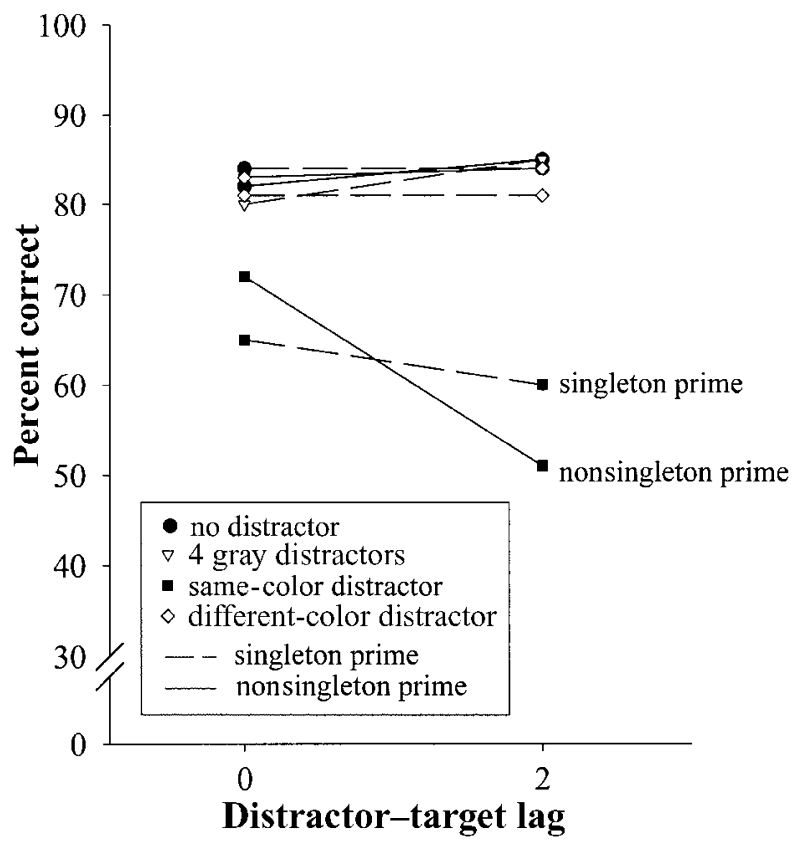

Figure 4. Mean percentages of correct target identification as a function of distractor condition, distractor-target lag, and prime status in Experiment 3.

ous experiments, distractor-target lags were limited to 0 and 2 . Given these design constraints, the experiment consisted of a total of 640 trials, in addition to 24 practice trials. Finally, each item in the RSVP sequence was presented for $50 \mathrm{msec}$, with a 50-msec ISI.

\section{Results}

The mean percentages of correct target identifications as a function of distractor condition, distractor-target lag, and prime status, collapsed across target color, are presented in Figure 4. The four different distractor conditions (i.e., no distractor, four-gray distractor, same-color distractor, and different-color distractor) are represented by the same symbols as in the previous figures, and prime status is represented by line type (dashed lines indicate that the prime was the singleton, solid lines indicate that the prime was a nonsingleton). For analysis purposes, trials in all four distractor conditions were assigned a lag value and a prime status (i.e., singleton vs. nonsingleton), even though both of these parameters were meaningless in the no-distractor condition and prime status was meaningless in the four-gray distractor condition. The data were subjected to a mixed ANOVA with target color the single between-subjects variable and distractor condition, distractor-target lag, and prime status the within-subjects variables.

The main effect of target color was significant, with better performance for red targets $(83.4 \%)$ than for green targets $\left[71.8 \% ; F(3,90)=51.80, M S_{\mathrm{e}}=754.6, p<.01\right]$. The main effect of distractor condition was also significant $\left[F(3,54)=59.64, M S_{\mathrm{e}}=146.3, p<.001\right]$, as was the interaction between target color and distractor condition $\left[F(3,54)=4.32, M S_{\mathrm{e}}=146.3, p<.01\right]$. The latter effect re- flects a larger overall decrement in the same-color distractor condition for green targets than for red targets. Target color did not enter into any other significant interactions.

The most obvious aspect of Figure 4 is that, consistent with the previous experiments, the only condition in which distractor-target lag had any effect at all was in the samecolor condition (solid squares). This was confirmed by a significant interaction between distractor condition and distractor-target lag $\left[F(3,54)=14.61, M S_{\mathrm{e}}=77.8, p<\right.$ $.0001]$. Turning to the effects of the location of the prime (solid vs. dashed lines), Figure 4 shows that, as with distractor-target lag, only the same-color distractor condition produced any effect of prime status. The analysis yielded a significant interaction between prime status and distractor-target lag $\left[F(1,18)=6.88, M S_{\mathrm{e}}=47.0, p<\right.$ $.05]$. However, this interaction was qualified by a threeway interaction with distractor condition $[F(3,54)=6.02$, $\left.M S_{\mathrm{e}}=59.7, p<.01\right]$. Simple interaction comparisons of prime status and distractor-target lag were conducted for each distractor condition. Only the same-color distractor condition produced any significant effects. Here, the main effect of lag was significant $\left[F(1,19)=13.9, M S_{\mathrm{e}}=\right.$ $2.40, p<.01]$. In addition, the interaction between lag and prime status was highly significant $[F(1,19)=10.09$, $\left.M S_{\mathrm{e}}=1.24, p<.01\right]$. Specifically, as can be seen in Figure 4 , for the same-color distractor condition, the effect of lag was attenuated when the prime occurred at the singleton location, a result consistent with the notion that attention was shifted to the location of the singleton and thereby enhanced central target identification.

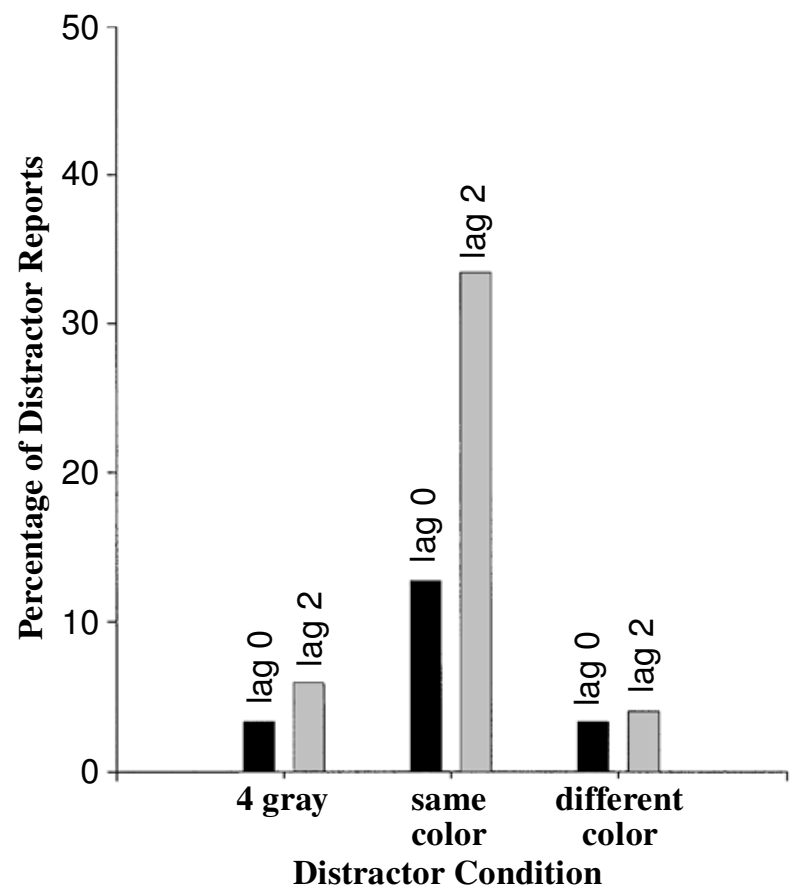

Figure 5. Mean percentages of distractor reports on error trials as a function of distractor condition and distractor-target lag in Experiment 3. 
As an additional check on this interpretation, we analyzed errors from the three conditions that contained distractors. On error trials, subjects report a letter other than the target letter in the central stream. We reasoned that if attention is shifted to the location of the singleton, on error trials the subjects should be biased to report the letter at the singleton location. The percentage of error trials on which the subjects reported the singleton letter instead of the central target letter is shown in Figure 5 for each of the three distractor conditions. As is clear from the figure, a disproportionately large percentage of errors in the same-color condition involved the report of the letter at the singleton location, and this is particularly evident at lag 2. An ANOVA of these data yielded significant main effects of distractor condition and lag $[F(2,36)=22.24$, $M S_{\mathrm{e}}=215.7, p<.001$, and $F(1,18)=28.06, M S_{\mathrm{e}}=68.7$, $p<.001$, respectively], as well as a significant interaction $\left[F(2,36)=19.21, M S_{\mathrm{e}}=63.78, p<.001\right]$. Focused comparisons yielded a significant effect of lag only in the same-color condition $\left[F(1,18)=38.29, M S_{\mathrm{e}}=112.4, p<\right.$ $.001]$.

\section{Discussion}

The results of the present experiment suggest that the decrement in central target identification associated with same-color distractors reflects a shift in spatial attention to the location of the singleton distractor. As in Experiment 2, only same-color distractors produced a decrement in central target identification. However, the effect of a same-color singleton was modulated by whether a prime character matching the central target appeared at the location of the singleton or not. Moreover, a large proportion of errors in the same-color distractor condition involved a report of the character at the singleton location. Together, these results are consistent with the spatial capture of attention by same-color singleton distractors.

However, one could argue that the priming effects observed in the present experiment reflect the selection of the prime by color rather than by spatial location (Duncan, 1981). That is, if the system is set to select and identify a red letter, perhaps any red letter in the display would gain access to response mechanisms, regardless of whether spatial attention is drawn to the location of that letter. Note that this account might still be considered a form of contingent attentional capture, in that spatially irrelevant stimuli are selected when they match top-down attentional control settings. However, it would suggest that the present results do not really speak to the issue of whether spatial attention can be captured when spatial uncertainty is eliminated. One way to rule out this alternative account would be to show priming effects for a character that appears at the location of a same-color distractor but that does not itself share the target color.

\section{EXPERIMENT 4}

Experiment 4 was conducted to test whether the priming effects observed in Experiment 3 reflect shifts of spa- tial attention to the location of the same-color distractor. The design was similar to that in Experiment 3, except that the distractors were now four boxes, one of which (i.e., the singleton distractor) was either red or green in the sameand different-color distractor conditions. In the frame immediately following the box distractor display, four gray peripheral letters appeared, one of which was a prime for the central target on that trial. The location of the prime relative to the preceding singleton box distractor was systematically manipulated across trials.

If spatial attention is drawn to the location of the samecolor box distractor, a subsequent gray prime appearing at that location should be identified and, therefore, enhance central target identification. If, however, the same-color distractors are selected purely on the basis of color without eliciting a shift of spatial attention to their location, a subsequent prime that does not share the target color

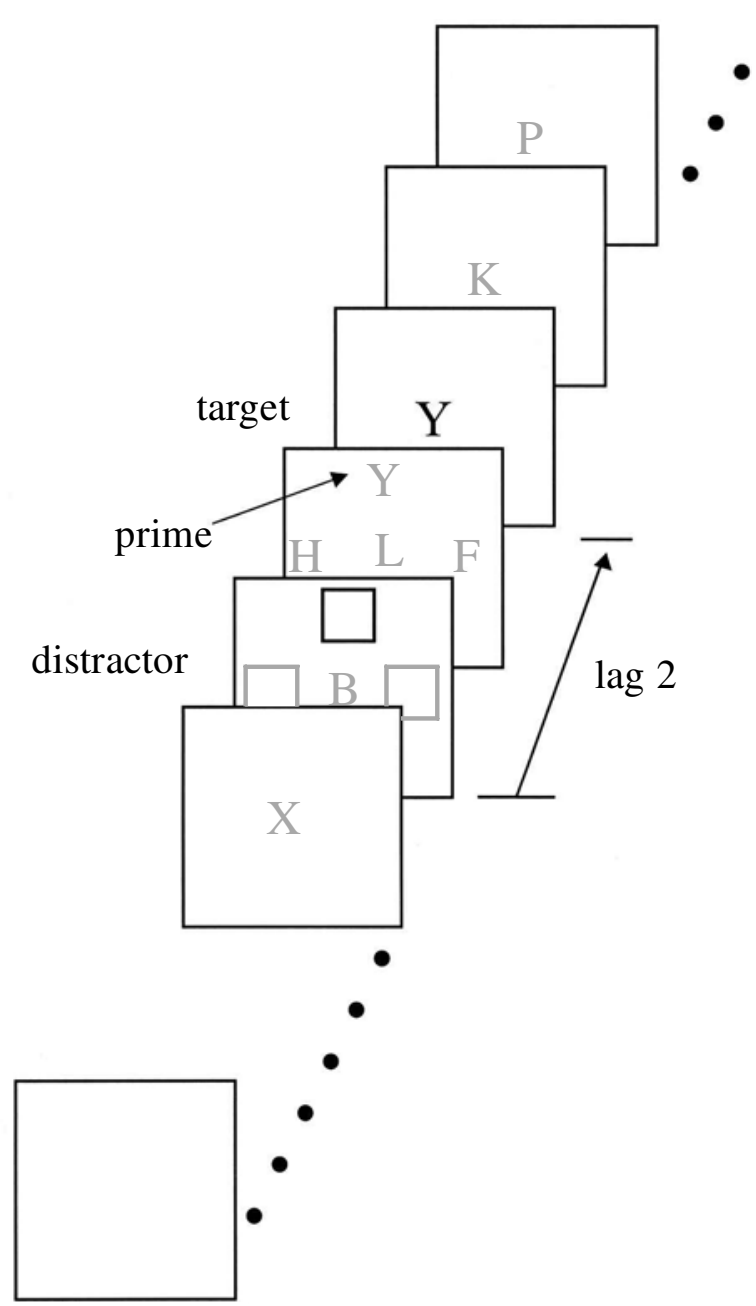

Figure 6. Representation of stimuli and sequence of events for Experiment 4. In this example, the distractor-target lag is 2, and the prime appears at the color singleton location. The boxes and characters printed in black were actually red or green (see the text). 
should not be selected and should, therefore, have no effect on central target identification.

\section{Method}

Subjects. Thirty new undergraduate students from Villanova University participated in this experiment. All had self-reported normal or corrected-to-normal visual acuity and color vision. Fifteen subjects searched for a red target, and 15 searched for a green target.

Apparatus and Stimuli. The apparatus and stimuli were identical to those used in Experiment 2.

Design and Procedure. The design and procedure were identical to those in Experiment 3, except that on each trial in the fourgray, same-color, and different-color distractor conditions, two peripheral events occurred rather than one (see Figure 6). The first event was the appearance of a box $\left(4.5^{\circ} \times 4.5^{\circ}\right.$, with a stroke width of $0.7^{\circ}$ ) at each of the four possible peripheral locations. Depending on the distractor condition, either all the boxes were gray or one of the boxes was red or green (i.e., same or different color from the central target). In the condition involving color singletons, the singleton box appeared equally often at each location across trials. Distractor-target lag was coded with respect to the appearance of these distractor boxes. The second peripheral event occurred on the frame immediately following the first event and consisted of the appearance of four gray letters, one at each of the four peripheral locations. As in Experiment 3, one of these letters (the prime) was always the same as the central target for that trial, and the three other letters were each chosen randomly without replacement from the remaining letters of the alphabet (excluding I, O, W, and Z). In the same-color and different-color distractor conditions, the prime appeared at the preceding color singleton location on one fourth of the trials. On no-distractor trials, no peripheral events occurred, although once again these trials were dummy-coded for both distractortarget lag and prime status.

\section{Results}

The mean percentages of correct target identifications as a function of distractor condition, distractor-target lag, and prime status are presented in Figure 7. The data were subjected to a mixed ANOVA with target color the single between-subjects variable and distractor condition, distractor-target lag, and prime status the within-subjects variables. The main effect of target color was not significant, nor did target color enter into any interactions. Distractor condition and distractor-target lag both produced significant main effects $\left[F(3,84)=39.65, M S_{\mathrm{e}}=9.70, p<\right.$ .0001 , and $F(1,28)=6.93, M S_{\mathrm{e}}=12.7, p<.01$, respectively]. These two variables also entered into a significant interaction $\left[F(3,84)=16.96, M S_{\mathrm{e}}=8.9, p<.0001\right]$. As is evident in the figure, this interaction is again driven by a deficit in performance that is specific to lag 2 in the same-color distractor condition.

Considering now the influence of the peripheral prime on central target identification, prime status produced a significant main effect $\left[F(1,28)=4.51, M S_{\mathrm{e}}=9.7, p<\right.$ $.05]$ and entered into a significant interaction with distractor condition. Simple effects analyses of prime status at each distractor condition yielded a significant effect in the same-color condition only $\left[F(1,28)=7.68, M S_{\mathrm{e}}=28.1\right.$, $p<.01]$. In this condition, gray primes at the singleton location produced an increase in accuracy of central target identification, relative to trials on which primes appeared at a non-singleton location. Interestingly, unlike Experiment 3, the effect of prime status did not interact

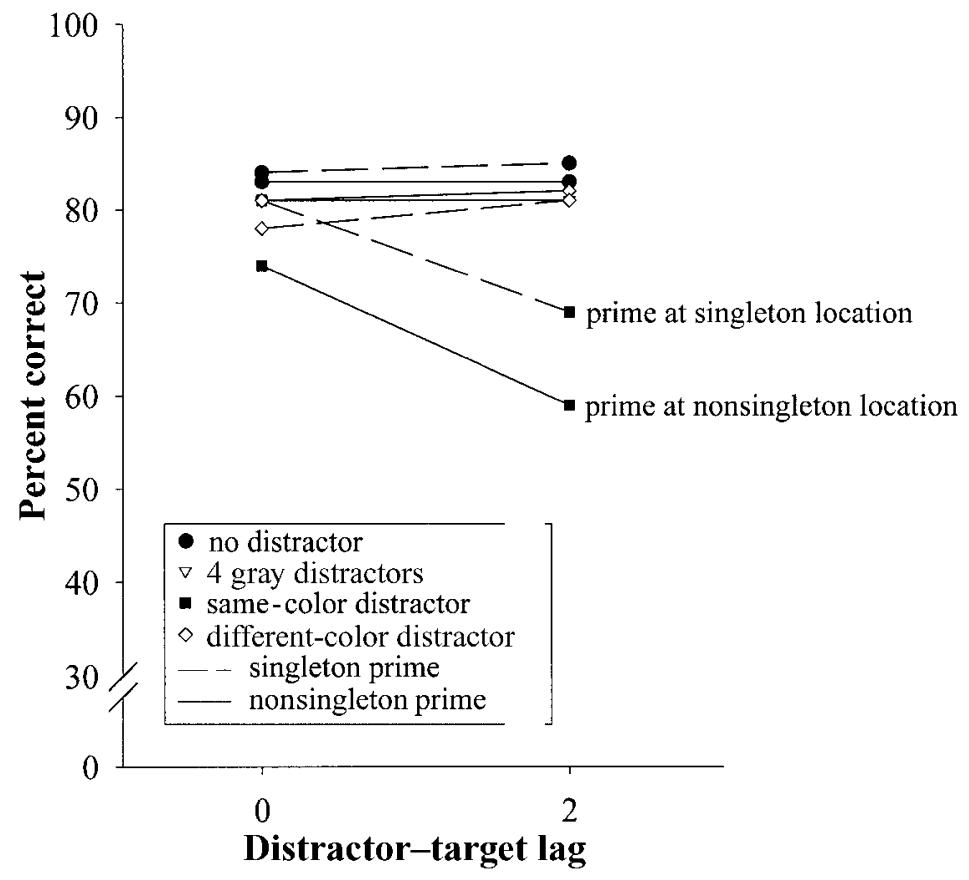

Figure 7. Mean percentages of correct target identification as a function of distractor condition, distractor-target lag, and prime status in Experiment 4. 


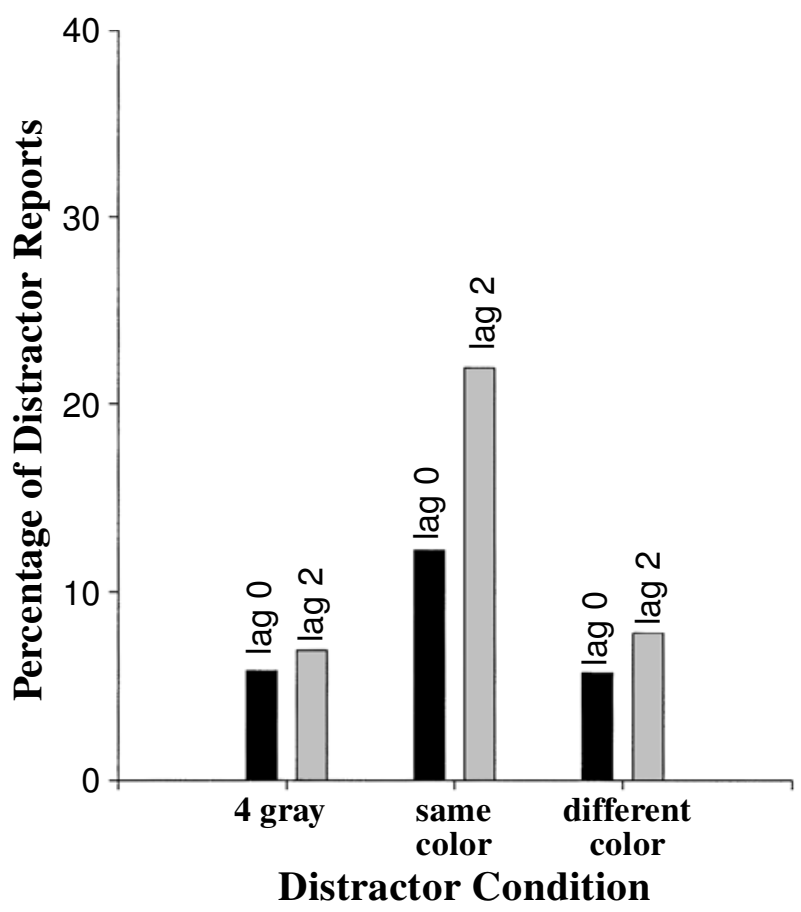

Figure 8. Mean percentages of distractor reports on error trials as a function of distractor condition and distractor-target lag in Experiment 4.

with target-distractor lag; there was no significant difference in the magnitude of the priming effect between lags 0 and 2. At this time, we have no explanation for this difference between the experiments.

An analysis of error trials from the three conditions that contained distractors yielded the same pattern as that in the previous experiment. The percentage of error trials on which the subjects reported the singleton letter instead of the central target letter is shown in Figure 8. Again, a disproportionately large percentage of errors in the samecolor condition involved the report of the letter at the singleton location, and this is particularly evident at lag 2 . An ANOVA yielded significant main effects of both distractor condition and lag $\left[F(2,56)=7.45, M S_{\mathrm{e}}=297.1, p<\right.$ .01 , and $F(1,28)=4.57, M S_{\mathrm{e}}=183.0, p<.05$, respectively]. Although the interaction between distractor condition and target-distractor lag just failed to reach significance, focused comparisons yielded a significant effect of lag in the same-color condition only $[F(1,28)=11.73$, $\left.M S_{\mathrm{e}}=122.02, p<.01\right]$.

\section{Discussion}

The results of this experiment essentially replicate those of the previous experiment. First, only the samecolor condition produced significant declines in central target detection. Second, this effect was modulated by whether a subsequent prime appeared at the location of the distractor. Third, a disproportionately large percentage of errors in the same-color condition involved the re- port of the letter at the singleton location, and this effect increased significantly with lag. However, in the present experiment, the letters in the periphery never appeared in the color of the central target (they were always gray). Instead, the effect of peripheral letters was specific to the spatial congruence between the same-color distractor and the subsequent letter. This pattern provides strong evidence that at least part of the central target decrement associated with same-color distractors reflects a shift of spatial attention to the location of the distractor. Of course, it is possible that a portion of the decrement may also be attributable to the traditional AB. However, for the present purposes, we have established that top-down attentional control settings can drive the capture of spatial attention even under focused attention conditions.

\section{GENERAL DISCUSSION}

Previous studies suggest that when the location of a target is certain, irrelevant stimuli are no longer capable of producing attentional capture (Theeuwes, 1991; Yantis \& Jonides, 1990). The present experiments were designed to determine whether this conclusion holds when the information about the location of a target does not eliminate uncertainty regarding which object at that location is the target. In addition, the studies were designed to explore whether capture under such conditions might be contingent on the match between the defining property of the target (i.e., the property used to eliminate nonspatial uncertainty) and the defining property of the irrelevant peripheral distractor.

The present experiments provide clear evidence that the spatial focusing of attention is not sufficient to eliminate the capture of spatial attention. In all the experiments, the appearance of an irrelevant color singleton outside the focus of attention produced a substantial decrement in central target identification. Moreover, the fact that this effect was specific to the match between the color of the irrelevant distractor and the color of the central target (Experiments 2-4) confirms that capture was contingent on top-down attentional control settings. Finally, the spatially specific influence of peripheral primes (Experiments 3 and 4) confirms that the effect of same-color peripheral distractors reflects spatial attentional capture, rather than simply constraints on temporal selection (i.e., the traditional $\mathrm{AB})$.

There are, however, several alternative accounts of these results that must be addressed. First, it is possible that the subjects made saccadic eye movements to the location of the singleton distractor. If so, given that the distractors appeared at $5^{\circ}$ eccentricity, the decrement in performance for the central target may simply reflect an associated decline in visual acuity. We consider this account unlikely. Because the distractor is completely irrelevant to the task, there is no incentive for subjects to voluntarily move their eyes to the distractor location. In fact, there would be a strong disincentive to do so if central target identification was com- 
promised. Of course, it is possible that the distractor may elicit reflexive saccades. However, although there is good evidence for reflexive saccades to irrelevant abrupt onsets (e.g., Theeuwes, Kramer, Hahn, \& Irwin, 1998), we know of no instances in the literature where reflexive eye movements have been elicited by irrelevant color singletons.

Nonetheless, as a check on this possibility, we ran a group of 6 subjects through the second experiment and obtained a rough measure of eye movements $\left(>1^{\circ}\right.$ to the right or left) with an Applied Science Laboratories Model 210 Eye-Trac eye movement monitor. The frequency of eye movements was no greater for trials on which irrelevant distractors appeared than on no-distractor trials. Moreover, when eye movement trials were excluded from the analysis, the pattern of results was the same as that in Experiment 2. Thus, we are confident that the decrement in target identification observed in the first two experiments is indeed an attentional effect, rather than sensory decline owing to eye movements.

A second alternative account of the present results is related to our assumption that the subjects were spatially focused on the central RSVP stream. One might argue that instead of being focused at fixation, the subjects' attention may have been in a more distributed state than they were, for example, in the cuing paradigms used by Yantis and Jonides (1990) and Theeuwes (1991). Thus, perhaps the reason same-color peripheral distractors appeared to capture attention is because they fell within the distributed "beam" of spatial attention. If this was the case, our conclusions about attentional capture under focused attention conditions would be undermined.

Although our experiments contained no direct manipulations that would address this issue, there are a number of important aspects of our design and data that render this possibility highly implausible. First, the physical dimensions of our displays would require that attention be distributed over an area nearly $10^{\circ}$ in diameter. Second, given the design of the experiments, there is very little incentive for the subjects to distribute attention, rather than focus on the central stream. The imperative target stimulus never appeared in the periphery, and potentially distracting stimuli appeared in the periphery on three fourths of all the trials. Thus, there is little reason to suspect that the subjects would try to distribute their attention, rather than remaining focused on the central stream. In fact, given that same-color peripheral distractors produced a significant decrement in performance, one could argue that there is a clear disincentive to do so. ${ }^{6}$ Third, performance on the central target identification task is well below ceiling, even in the no-distractor conditions (i.e., it hovers around $80 \%$ correct). Thus, the difficulty of the central task clearly places a high demand on processing resources, making it unlikely that there are attentional resources that are "left over" to be distributed in the periphery (Lavie, 1995). Finally, the last two priming experiments provide an indirect opportunity to look for evidence of distributed attention. One might suspect that if attention were distributed, priming effects might be apparent in any condition in which a prime appears in the periphery, such as the four-gray and different-color distractor conditions. This prediction is based on the assumption that if a stimulus appears within the beam of attention, its identity will be processed and will influence central target detection (an assumption supported by results of the same-color distractor conditions of the priming experiments). However, only the same-color distractor condition produced any evidence of priming; the presence of peripheral primes in the other conditions had absolutely no effect on performance. ${ }^{7}$

One might still argue, however, that perhaps the subjects had difficulty maintaining attentional focus over the course of a trial, resulting in the "wandering" of spatial attention. As in the previous case, this scenario would undermine our assumption that attention was tightly focused at fixation. One way of addressing this possibility is to take advantage of the fact that the temporal position of the target in the central stream varied across trials. That is, on some trials the target appeared relatively early in the sequence, and on others, relatively late. If subjects have difficulty maintaining attentional focus over the course of a trial, we might expect performance to drop as the target appears later and later in the sequence. A quick glance at the data from the no-distractor conditions of Experiments 1 and 2 provides no support for such an effect. In Experiment 1, the mean performance for target positions $8-12$ in the 18 -letter sequence was $.79, .80, .80, .80$, and .82 , respectively. For Experiment 2, the corresponding numbers were $.83, .86, .87, .85$, and .84 . The fact that performance remains relatively constant across target positions suggests that attention did not, in fact, wander from the central stream. In summary, we contend that the logic of the experimental design, as well as the results obtained, make it highly unlikely that the subjects were in anything other than a focused attention state.

\section{Implications for Attentional Control}

In addition to the showing that spatial certainty is not sufficient to eliminate attentional capture, the results of these experiments have a number of implications for theories of attentional control and may also have some important implications for the AB phenomenon. With respect to attentional control, the results provide converging evidence for the contingent involuntary orienting hypothesis. Previous studies of contingent orienting have used response time differences as a function of the spatial relationship between precue and target to index influence of top-down set on the capture of attention. The present experiments show further evidence of contingent orienting, using accuracy in an RSVP paradigm. The results also extend our understanding of the nature and influence of top-down attentional control settings. Specifically, these experiments suggest that an attentional control setting for resolving nonspatial uncertainty can also impact the deployment of spatial attention. An alternative way of thinking about this issue is that the subjects were unable to adopt a conjunctive control setting consisting of the spatial location (i.e., fixation) and color (e.g., red), that would have restricted attentional selection to the target. Selection by color, which was critical to target acqui- 
sition, could not be conditionalized on the subjects' set for location. Thus, stimuli that shared the critical color captured attention even when they occurred outside the focus of attention at an irrelevant location (related results have recently been reported by Zuvic, Visser, \& Di Lollo, 2000).

Finally, the comparison of the results of Experiments 1 and 2 provide further evidence for the distinction between singleton search mode and feature search mode introduced by Bacon and Egeth (1994). The fact that these two levels of attentional set are possible suggests that topdown control settings are quite flexible and can be instantiated at different levels in the system (e.g., at the level of specific color values or at the level of color singletons in general). In addition, Experiment 1 provides an interesting insight into the nature of singleton search mode. Color singleton distractors were defined by a difference in color across space (i.e., a single color \# among three gray \#s). The central target, however, was a singleton only with respect to changes in color over time. This finding suggests that for the purposes of attentional control, spatial and temporal singletons are functionally equivalent.

\section{Implications for the Attentional Blink}

The results of the present experiments may also have implications for the $\mathrm{AB}$ phenomenon. In previous work, the $\mathrm{AB}$ was observed in a dual-task situation in which responses were required for both $\mathrm{T} 1$ and $\mathrm{T} 2$. In fact, the $\mathrm{AB}$ is typically measured relative to a control condition in which T1 is ignored. However, the present results show that a spatial blink can be produced by an ignored, irrelevant distractor that appears outside the central stream and for which no response is required. This opens up the interesting possibility that the traditional $\mathrm{AB}$ may also be produced by a $\mathrm{T} 1$ for which no response is required, as long as the $\mathrm{T} 1$ shares the defining property of T2. In fact, there are hints of just such effects in the existing AB literature (Chun, 1997; Ward, Duncan, \& Shapiro, 1997). For example, Chun found that $\mathrm{T} 1$ produced a significant decrement in $\mathrm{T} 2$ report even in control conditions in which T1 was ignored. In the Chun study, each item in the sequence was surrounded by a white outline square, except for $\mathrm{T} 1$ and $\mathrm{T} 2$, which were defined by colored outline squares. Thus, subjects may have been "set" to select the target on the basis of a color singleton, which, according to our speculations, may have resulted in contingent temporal capture by an otherwise ignored $\mathrm{T} 1$ (which was also defined by a color singleton). A similar effect can be found in Ward et al., (1997, Experiment 1), who used a skeletal RSVP task in which only masked T1 and T2 items were presented (i.e., there were no intervening distractor items). The control conditions in which $\mathrm{T} 1$ was ignored yielded an approximate 20\% drop in T2 report at short T1-T2 SOAs. Although this effect was not directly tested statistically, nor was it as large as when T1 was reported, it is consistent with the notion that T1 may have produced some degree of contingent capture, based, perhaps, on the fact that both $\mathrm{T} 1$ and $\mathrm{T} 2$ were relatively isolated abrupt onsets (i.e., they were defined by similar properties). Thus, the small SOA effects in the control conditions of the traditional $\mathrm{AB}$ may indeed reflect a form of temporal contingent attentional capture. If so, this may have important implications with respect to measuring the magnitude of the $\mathrm{AB}$, as well as with respect to theoretical interpretations of the $\mathrm{AB}$. We are currently exploring this possibility (related work has recently been reported by Hillstrom, Chai, \& Leeman, 2000).

There is one particularly interesting aspect of the present results that is also relevant to the $\mathrm{AB}$ literature. In the first two experiments, the greatest disruption to target identification occurred at lag 2; when the target appeared at lag 1, the distractor had relatively little effect. This phenomenon is common in the $\mathrm{AB}$ literature and is referred to as lag 1 sparing. However, a recent analysis by Visser, Zuvic, Bischof, and Di Lollo (1999) suggests that lag 1 sparing should be evident only when both critical events occur at the same spatial location. They systematically manipulated whether T1 and T2 appeared at the same location (i.e., both central or both eccentric) or at different locations (i.e., $\mathrm{T} 1$ central and $\mathrm{T} 2$ eccentric, or vice versa). Lag 1 sparing was evident only when the two targets appeared at the same location. The authors proposed that lag 1 sparing is the result of a sluggish, spatially based, attentional gating system. Specifically, when T1 occurs, a spatial attention gate is opened quickly at the T1 location but closes more slowly, so that if T2 occurs soon after T1 in the same location, it will get passed on to subsequent processing stages, producing lag 1 sparing. When T2 appears at a different location, it is not passed through the spatially specific attentional gate that opened in response to $\mathrm{T} 1$, and thus no lag 1 sparing occurs.

In the present experiments, the distractor appeared at an eccentric location and the target at a central location, and yet there was still evidence for lag 1 sparing. At first glance, these results seem to conflict with those of Visser, Zuvic, et al. (1999). However, there is one critical difference between the two paradigms that leads to one possible (and relatively simple) resolution. In the spatial shift conditions of the Visser, Zuvic, et al. study, subjects began by monitoring the spatial location of the first critical event (i.e., T1). Under such conditions, lag 1 sparing does not obtain, because T2 appears before spatial attention has shifted to the location of T2. Now consider the analogous trials in the present experiments, in which the peripheral distractor appears prior to the target in the central stream. The subjects began by monitoring the location of the second critical event (i.e., the target in the central stream). Thus, any delay in the shift of attention to the distractor would produce lag 1 sparing if the target occurs during that delay (i.e., before attention has shifted away from the central stream).

What might produce such a delay in the shift of attention to the distractor? One possibility is that attentional disengagement from the central stream might be sluggish precisely because subjects are actively trying to attend to the central stream. Another possibility is based on the notion of prior entry (as described in Boring, 1957; Stelmach \& Herdman, 1991). Stelmach and Herdman, using temporal order judgments of visual stimuli, demonstrated a pro- 
cessing advantage of 40-50 msec for an attended stimulus, as compared with an unattended one. In our experiments, the distractor appeared at an unattended location and might, therefore, be subject to a delay in processing, relative to the attended stimuli in the central stream. Furthermore, in Stelmach and Herdman's work the two stimuli were equidistant from fixation, whereas in our experiments one stimulus was at fixation and the other was at $5.2^{\circ}$ eccentricity; this may contribute further to the processing delay of the distractor. Thus, although the distractor appears approximately $100 \mathrm{msec}$ before the target on lag 1 trials, the processing of the distractor (which presumably provides the signal to shift attention to the distractor location) may not be complete until after the target in the central stream has appeared, producing lag 1 sparing. Both of these scenarios are consistent with the notion that lag 1 sparing results from the sluggish closing of spatial attentional gates, as was proposed by Visser, Zuvic, et al. (1999; see also Visser, Bischof, \& Di Lollo, 1999).

\section{A Final Caveat}

We have argued on the basis of the present results that the focusing of spatial attention is not sufficient to eliminate attentional capture when in the presence of top-down control settings. However, we should point out that it is possible that the act of focusing spatial attention on an RSVP stream, as in the present experiments, involves a different process than the act of focusing attention in response to a spatial precue, as in the studies by Yantis and Jonides (1990) and Theeuwes (1991). In an RSVP task, even though subjects are attending to the location of the stream, only a subset of the information presented at that location is "selected" for response. This suggests that the act of attending to a location can be dissociated from the act of passing information at that location on to response or decision stages (see also Nakama \& Egeth, 1999; Remington \& Folk, 2001). Visser, Zuvic, et al. (1999) have suggested that the latter process in an RSVP task involves the opening of a spatial gate, which is driven by the occurrence of the defining property of the target. In the spatial precuing experiments, on the other hand, there is no need to withhold the opening of a spatial gate at the cued location, because the cue is $100 \%$ valid and the only stimulus that will occur at the cued location is the target. Thus, perhaps irrelevant peripheral stimuli can, in fact, capture attention when subjects are spatially attending to a location (as in the present experiments), but not after a spatial gate for selection has been opened (as might be the case in precuing studies). Clearly, further research is needed to explore the relationship between spatial selection and attentional capture.

\section{REFERENCES}

Bacon, W. F., \& Egeth, H. E. (1994). Overriding stimulus-driven attentional capture. Perception \& Psychophysics, 55, 485-496.

BorING, E. G. (1957). History of experimental psychology (2nd ed.). New York: Appleton-Century-Crofts.

Broadbent, D. E., \& Broadbent, M. H. P. (1987). From detection to identification: Response to multiple targets in rapid serial visual presentation. Perception \& Psychophysics, 42, 105-113.

Chun, M. M. (1997). Temporal binding errors are redistributed by the attentional blink. Perception \& Psychophysics, 59, 1191-1199.

Chun, M. M., \& Potter, M. C. (1995). A two-stage model for multiple target detection in rapid serial visual presentation. Journal of Experimental Psychology: Human Perception \& Performance, 21, 109-127.

Duncan, J. (1981). Directing attention in the visual field. Perception \& Psychophysics, 30, 90-93.

ERIKSEN, B. A., \& ERIKSEN, C. W. (1974). Effects of noise letters upon the identification of a target letter in a nonsearch task. Perception \& Psychophysics, 16, 143-149.

Folk, C. L., \& Remington, R. W. (1998). Selectivity in distraction by irrelevant featural singletons: Evidence for two forms of attentional capture. Journal of Experimental Psychology: Human Perception \& Performance, 24, 847-858.

Folk, C. L., Remington, R. W., \& Johnston, J. C. (1992). Involuntary covert orienting is contingent on attentional control settings. Journal of Experimental Psychology: Human Perception \& Performance, 18, 1030-1044.

Folk, C. L., Remington, R. W., \& Johnston, J. C. (1993). Contingent attentional capture: A reply to Yantis (1993). Journal of Experimental Psychology: Human Perception \& Performance, 19, 682-685.

Folk, C. L., Remington, R. W., \& Wright, J. H. (1994). The structure of attentional control: Contingent attentional capture by apparent motion, abrupt onset, and color. Journal of Experimental Psychology: Human Perception \& Performance, 20, 317-329.

Gibson, B., \& Kelsey, E. (1998). Stimulus-driven attentional capture is contingent on attentional set for display-wide visual features. Journal of Experimental Psychology: Human Perception \& Performance, 24, 699-706.

Hillstrom, A., Chai, Y., \& Leeman, S. (2000, November). Attention to objects that change identity. Paper presented at the 41 st Annual Meeting of the Psychonomic Society, New Orleans.

Lavie, N. (1995). Perceptual load as a necessary condition for selective attention. Journal of Experimental Psychology: Human Perception \& Performance, 21, 451-468.

NaKama, T., \& EgETh, H. E. (1999). The dependence of attentional blink on the temporal position of the target in RSVP. Investigative Ophthalmology \& Visual Science, 40, S49.

Pashler, H. (1988). Cross-dimensional interaction and texture and texture segregation. Perception \& Psychophysics, 43, 307-318.

Peterson, M. S., \& Juola, J. F. (2000). Evidence for distinct attentional bottlenecks in attention switching and attentional blink tasks. Journal of General Psychology, 127, 6-26.

Raymond, J. E., Shapiro, K. L., \& Arnell, K. M. (1992). Temporary suppression of visual processing in an RSVP task: An attentional blink? Journal of Experimental Psychology: Human Perception \& Performance, 18, 849-860.

Raymond, J. E., Shapiro, K. L., \& Arnell, K. M. (1995). Similarity determines the attentional blink. Journal of Experimental Psychology: Human Perception \& Performance, 21, 653-662.

Remington, R. W., \& Folk, C. L. (2001). A dissociation between attention and selection. Psychological Science, 12, 511-515.

Remington, R. W., Johnston, J. C., \& Yantis, S. (1992). Involuntary attentional capture by abrupt onsets. Perception \& Psychophysics, 51, 279-290.

Stelmach, L. B., \& Herdman, C. M. (1991). Directed attention and perception of temporal order. Journal of Experimental Psychology: Human Perception \& Performance, 17, 539-550.

Theeuwes, J. (1991). Exogenous and endogenous control of attention: The effect of visual onsets and offsets. Perception \& Psychophysics, 49, 83-90.

Theeuwes, J. (1992). Perceptual selectivity for color and form. Perception \& Psychophysics, 51, 599-606.

TheeuweS, J. (1994). Stimulus-driven capture and attentional set: Selective search for color and visual abrupt onsets. Journal of Experimental Psychology: Human Perception \& Performance, 20, 799-806.

Theeuwes, J., Kramer, A., Hahn, S., \& Irwin, D. E. (1998). Our eyes do not always go where we want them to go: Capture of the eyes by new objects. Psychological Science, 9, 379-385. 
Visser, T. A. W., Bischof, W. F., \& Di Lollo, V. (1999). Attentional switching in spatial and nonspatial domains: Evidence from the attentional blink. Psychological Bulletin, 125, 458-469.

Visser, T. A. W., Zuvic, S. M., Bischof, W. F., \& Di Lollo, V. (1999). The attentional blink to targets in different spatial locations. Psychonomic Bulletin \& Review, 6, 432-436.

Ward, R., Duncan, J., \& SHAPIRO, K. (1997). Effects of similarity, difficulty, and nontarget presentation on the time course of visual attention. Perception \& Psychophysics, 59, 593-600.

YANTIS, S. (1993). Stimulus-driven attentional capture and attentional control settings. Journal of Experimental Psychology: Human Perception \& Performance, 19, 676-681.

YAnTIS, S., \& Johnston, J. C. (1990). On the locus of visual selection: Evidence from focused attention tasks. Journal of Experimental Psychology: Human Perception \& Performance, 16, 135-149.

YANTIS, S., \& Jonides, J. (1984). Abrupt visual onsets and selective attention: Evidence from visual search. Journal of Experimental Psychology: Human Perception \& Performance, 10, 601-621.

YANTIS, S., \& JoNides, J. (1990). Abrupt visual onsets and selective attention: Voluntary versus automatic allocation. Journal of Experimental Psychology: Human Perception \& Performance, 16, 121-134.

Zuvic, S., Visser, T. A., \& Di Lollo, V. (2000, November). Contingent capture in a nonsearch task. Paper presented at the 41st Annual Meeting of the Psychonomic Society, New Orleans.

\section{NOTES}

1. It is important to emphasize that the focusing of attention in these experiments eliminated the capture of spatial attention by the distractor. The results do not necessarily imply that stimuli outside the focus of attention can never influence target processing. For example, there are many studies showing that the identity of irrelevant distractors that are spatially separated from the known location of a target can influence response time to the identity of the target (e.g., Eriksen \& Eriksen, 1974; but see also Yantis \& Johnston, 1990).

2 . We should note that some have argued that when a peripheral distractor shares the defining color of the target, it should no longer be considered "irrelevant." For a discussion of this and related issues, see the exchange between Yantis (1993) and Folk, Remington, and Johnston (1993).

3. Owing to a programming error, the $42-\mathrm{msec}$ interstimulus interval and stimulus duration values actually represent the mean of a sawtooth distribution with a minimum of $33.5 \mathrm{msec}$ and a maximum of $50.3 \mathrm{msec}$. Therefore, the total SOA from one letter to the next varied between $67 \mathrm{msec}$ ( $25 \%$ of the trials), $83.8 \mathrm{msec}$ ( $50 \%$ of the trials), and $100.6 \mathrm{msec}$ ( $25 \%$ of the trials). However, this distribution was consistent across all distractor conditions.

4. As in Experiment 1, the stimulus duration actually varied according to a sawtooth distribution, this time with a minimum of $50 \mathrm{msec}$, a maximum of $67 \mathrm{msec}$, and a mean of $56 \mathrm{msec}$. Thus, the SOA between letters varied from $84 \mathrm{msec}$ ( $32.6 \%$ of the trials) to $100 \mathrm{msec}(50.1 \%$ of the trials) to $117 \mathrm{msec}$ ( $17.3 \%$ of the trials). Once again, these distributions were consistent across all distractor conditions.

5 . The programming error in the first two experiments was corrected in all the subsequent experiments.

6 . We should also point out that our focusing assumption is similar to the assumption, in visual search studies of attentional capture, that uncorrelating the location of the target and the location of the capturing stimulus property removes any incentive to voluntarily shift attention to the capturing property.

7. Note that the lack of priming effects in the different-color condition also provides evidence against the possibility that the subjects voluntarily allocated attention to the singleton distractor. One might argue that if subjects believe the peripheral singleton heralds a targetmatching prime on some trials, they may voluntarily allocate resources to the singleton to enhance performance. However, given that distractor types were mixed within blocks, this account predicts prime status effects in the different-color distractor condition as well.

(Manuscript received September 13, 2000; revision accepted for publication October 17, 2001.) 